\title{
The Analysis of Training Idea of Adolescent Wushu based on the New Rule of Competitive Wushu Routine
}

\author{
Yong Ding, Shuai Liu \\ Pingxiang University, Pingxiang, Jiangxi, 337055, China
}

\section{Keywords: New rules; Wushu routine; adolescent training}

\begin{abstract}
Based on the implementation of Wushu new rules and the development trend of the current competitive Wushu, this article emphasizes the importance of the strength and speed training of Wushu routines of the adolescent, and further puts forward the corresponding training methods and means. The new rule is not only the ruler of the athlete to judge the technical level and athletic ability, but also an important basis for the training of coaches. It leads the direction of technological development, and at the same time, affects the youth martial arts routine training. A substantial, jumping change is carried out in the scoring method and the content of the score, especially in the optional routines and scoring methods on the content of the drastic reform, which break the unique Chinese culture. The most prominent thing is that the optional item adds a score for the difficulty action. Difficult movements (including the difficulty of movement and connection difficulty and the difficulty of throwing the instrument) make the value orientation of martial arts routine changing.
\end{abstract}

\section{Introduction}

The implementation of the new rules leads the development of competitive martial arts to a high, difficult, beautiful and new direction. It is more consistent with the competition rules of the Olympic Games. It is a good trend and beginning of the Chinese Wushu walking to the world [1-3]. There is no doubt that the strength and speed training of young Wushu athletes put forward higher requirements. The previous training methods and means can not fully meet the needs of the current development of competitive Wushu. Grasping the strength and speed training of young athletes is the guarantee to obtain excellent results in training.

The Chinese martial arts source flows for long. Athletic Wushu routine sports as a traditional Chinese sports has a long history. The contest rule is established for athletic contest of unify the norm with standard, since it is an umpire to adjudicate the athlete technique level with the surveyor rod that contests the ability. The coach member that is also an importance trains basis [4, 5]. The evolution of the new rules guides the Wushu routine sports technology innovation. In particular, the new rules increase the Wushu routine difficulty of the coefficient and score. An increase of a variety of different groups of balanced action highlights the action specifications. Increase the proportion of the score, and enhance the level of exercise scores. Routine athletes have a higher level of technical requirements.

A substantial, jumping change is carried out in the scoring method and the content of the score, especially in the optional routines and scoring methods on the content of the drastic reform, which break the unique Chinese culture. The most prominent thing is that the optional item adds a score for the difficulty action. Difficult movements (including the difficulty of movement and connection difficulty and the difficulty of throwing the instrument) make the value orientation of martial arts routine changing [6]. Rules as competition and training norms and guidelines, its evolution and technology development are closely related. The strength training of young Wushu athletes should be based on the characteristics of the technology (for example, the strength of the practice and the difficulty of action structure and technical action of the force characteristics should be very similar). Focusing on the upper limbs and trunk parts and the development of spring force, speed strength training. Remind: the strength of young athletes practice to strictly control the weight and duration of the load, so as not to affect the normal growth of bones. 


\section{The principles of training for adolescent}

Adolescence is the stage of the "qualitative change" of the organism in the process of growth and development. At this stage, the tissues and organs of adolescents have great development potential and plasticity in structure and function. In the training, arrangement reasonably and follow the principles of scientific training.

First of all, the old rules stipulate that the optional items must be selected for a certain number of steps, hand movements, balanced jumps, and instrumental methods for different groups. The new rule does not have this limitation. In other words, the new rules which include the difficulty of action 2 points action do not limit the composition of the Wushu routine and basic skills of the action group. Athletes who are in the optional project in addition to the difficulty of action routines can choose any action. In contrast, the basic steps, footwork, hand, technique, and instrumental methods that have been made up of Wushu routines have been diluted. Second, the new rules are in the selection of the contents of the changes in the score. So the difficulty of action in the optional items highlights the significance.

\subsection{The combination of basic training and special technology principle}

In the Wushu training of young people, pay attention to the overall development of the body according to the characteristics of young people's physiological and psychological characteristic. That is, through systematic scientific training their body are fully developed which is engaged in special technology. And this is the material basis of training. On the basis of the overall development of the body, combine of special technical requirements, training methods and action specifications to train, in order to achieve mastery of technical action and improve athletic performance.

\subsection{Step by step principle}

Suitable exercise load refers to the physical and psychological characteristics of the young people during training. The size of the load has an important effect on the training effect. The weak load can not cause the change of the body function, and the excessive load is detrimental to health. For a person, the exercise load is not fixed. With the improvement of the ability to adapt to the human body, the load should be from small to large. And give the body a new stimulus gradually, so that the body can continue to maintain the trend of growth.

\subsection{Strength training methods and content}

The strength training of young Wushu athletes should be based on the characteristics of the technology (for example, the strength of the practice and the difficulty of action structure and technical action of the force characteristics should be very similar). Focusing on the upper limbs and trunk parts and the development of spring force, speed strength training. Remind: the strength of young athletes practice to strictly control the weight and duration of the load, so as not to affect the normal growth of bones.

\section{The evolution of the new rules guided the innovation of Wushu routine}

\subsection{The value highlighting of the difficulty of action}

First, the changes are in the selection of the optional items in the new rules. The new rules appear in the difficulty of the action of the score, optional items of the entire exercise level and action specifications to reduce the proportion. The whole score system is based on the overall quality of action (specifications) and exercise level. The rule in 1996 began to integrate the spirit of coordination and spirit, rhythm, style, content, structure, and lay the two scores into a level of exercise $[7,8]$. This part is the embodiment of the martial arts, on the one hand, is the most vague score content. At this time there is no difficulty in the rules of action points. The difficulty of action is only to give the overall consideration to score in the project content, structure and layout. So the some 
athletes choose some actions which they are much sure and not easy to deduct the scores in order to improve the atmosphere of the entire project In fact few people are interested in 0.2 innovation difficulty points.. The new rules in 2014 further reduced the quality of the action and the level of exercise points, and increased the score of the difficulty of action (see in Table 1). The new rules increase the difficulty of the action, the difficulty of the movement, the difficulty of the connection and the difficulty of quantification. The athletes in the whole set of actions must be selected for a certain score of the difficulty of action. The difficulty of selecting the action score is not enough. Difficulty action to complete the quality does not meet the rules required, and there is no difficulty score. So the difficulty of action in the full set is particularly important.

Table 1. Score distribution rules in recent years

\begin{tabular}{c|c|c|c|c|c|c}
\hline year & content & score & content & score & content & score \\
\hline 2005 & $\begin{array}{c}\text { action } \\
\text { specification }\end{array}$ & 6.00 & $\begin{array}{c}\text { strength and } \\
\text { coordination }\end{array}$ & 2.00 & $\begin{array}{c}\text { Spirit, } \\
\text { cadence, } \\
\text { structure, } \\
\text { style }\end{array}$ & 2.00 \\
\hline 2010 & $\begin{array}{c}\text { action } \\
\text { specification }\end{array}$ & 6.80 & $\begin{array}{c}\text { Performance } \\
\text { level }\end{array}$ & 3.00 & $\begin{array}{c}\text { Innovation } \\
\text { difficulty }\end{array}$ & 0.20 \\
\hline 2015 & Action quality & 5.00 & $\begin{array}{c}\text { Performance } \\
\text { level }\end{array}$ & 3.00 & $\begin{array}{c}\text { Element of } \\
\text { difficulty }\end{array}$ & 2.00 \\
\hline
\end{tabular}

\subsection{The proportion of basic technology is weakened}

First of all, the old rules stipulate that the optional items must be selected for a certain number of steps, hand movements, balanced jumps, and instrumental methods for different groups. The new rule does not have this limitation. In other words, the new rules which include the difficulty of action 2 points action do not limit the composition of the Wushu routine and basic skills of the action group. Athletes who are in the optional project in addition to the difficulty of action routines can choose any action. In contrast, the basic steps, footwork, hand, technique, and instrumental methods that have been made up of Wushu routines have been diluted. Second, the new rules are in the selection of the contents of the changes in the score. So the difficulty of action in the optional items highlights the significance.

According to the new rules in the exercise level of the three grades nine sub-levels principle, each file difference is 0.4 points, and stalls empty 0.1 points, which means each stall only difference of 0.1 points (see in Table 2 ). And the minimum score of a single difficulty action also have 0.2 points. Such as a C-level action needs D-class connection up to 0.6 points. However, 0.2 points which are related to the action specifications and exercise level are concerned, and it is a secondary span. Compared with the exercise level score method is still high. Difficult action, especially the difficult situation to complete the situation to the 2014 rules, for example, a "C-level action difficulty + D-level connection" score, is 0.6 points. In contrast, the difficulty of the action in the game scoring efficiency is much higher than the level of exercise, and it needs time and effort to improve the level of exercise training. 
Table 2. The criteria for the level of exercise in 2014

\begin{tabular}{ccc}
\hline Three grades & Nine levels & 2014 \\
\hline \multirow{3}{*}{ Excellent } & First level & $3.00-2.90$ \\
& Second level & $2.89-2.80$ \\
& Third level & $2.79-2.70$ \\
\multirow{2}{*}{ Common } & Forth level & $2.60-2.50$ \\
& Fifth level & $2.49-2.40$ \\
& Sixth level & $2.39-2.30$ \\
Worth & Seventh level & $2.10-2.00$ \\
& Eighth level & $1.99-1.90$ \\
& Ninth level & $1.89-1.80$ \\
\hline
\end{tabular}

\subsection{Comprehensive and balanced development of physical fitness training}

In the past, the kind method of avoiding the other side of the short arrangement, due to the introduction of difficulty scores, is not applicable. Now Wushu set line athletes must have a very comprehensive physical quality, in order to ensure the completion of these difficult moves and innovation. The difficulty of Wushu project is classified by the type of action. It is divided into the balance, the action of legs difficulty; jump, flutter action difficulty; connection action and throwing difficulty. Different types of action have different requirements to the physical quality. Such as balance, leg method is difficult to move the quality of flexibility. Jump, flutter movement is difficult to jump the main quality of the jump. Connection action and throwing the difficulty is mainly stable. But the completion of each difficulty is not a certain quality of independent performance. It is a comprehensive response to the quality of multiple.

For example: "Tornado feet 540 degrees splicing" of the complete action, it requires athletes having excellent spring jump, good flexibility, good stability, rapid rotation in the vacated state, and landing when the balance to complete the stability of the capacity, and so on a variety of qualities. The completion of each action is a variety of athletes overall physical performance. Therefore, the youth training should seize the development of children's physical fitness sensitive period. The overall development of the general physical fitness and special physical fitness create the conditions for the development of difficult action. The basic training of adolescents must strengthen the physical and ability training related to the difficulty of action. From the difficulty of improving the exercise to the exercise state, and then integrated into the entire routine. So that the whole action quality, exercise level, difficulty overall development.

First of all, the old rules stipulate that the optional items must be selected for a certain number of steps, hand movements, balanced jumps, and instrumental methods for different groups. The new rule does not have this limitation. In other words, the new rules which include the difficulty of action 2 points action do not limit the composition of the Wushu routine and basic skills of the action group. Athletes who are in the optional project in addition to the difficulty of action routines can choose any action. In contrast, the basic steps, footwork, hand, technique, and instrumental methods that have been made up of Wushu routines have been diluted. Second, the new rules are in the selection of the contents of the changes in the score. So the difficulty of action in the optional items highlights the significance.

\section{Summary}

The new rules, while highlighting the difficulty of Wushu routines, weaken the most basic technical content of Wushu routine movement. So that young people tend to focus on training difficulties and difficulties associated with the physical training. Rules as training policy guidance, in guiding Wushu routine movement towards the development of competitive at the same time, cannot abandon the Wushu routine unique basic methods and techniques. We must retain the essential characteristics of Wushu. So that Wushu routine movement toward a more rational, more scientific direction. The aim is investigating the development trend of the current new rule in orientation and 
tournament Wushu gradually to synthesize the training method homogonously with the means. And provide with the training of the athlete for the all levels.

\section{References}

[1] Leonetti D L, Chabot-Hanowell B. Competitive Wushu Routine Training after the Implementation of New Rules of Wushu Routine Competitions[J]. Wushu Science, 2011, 117(9):2073-8.

[2] Li Z, Xie F. On the Teaching Steps and Methods for Difficult Movement of Competitive Wushu Routine[J]. Wushu Science, 2014.

[3] Mo-Zhu L V. Analysis on Technical Development of Excellent Chinese Wushu Routine Athletes in the New Rule[J]. China Sport Science \& Technology, 2010.

[4] Zhou X Y. Research on the Development of Multimedia CAI Course Software of "The National Wushu Routine Competition Rules and Judgment"[J]. Journal of Beijing Sport University, 2006.

[5] Xi B. The Swot Analysis on the Reserved Talents Training System of Competitive Wushu Routine in Jiangsu Province[J]. Wushu Science, 2013.

[6] Zhao X. On the Structure and Training of Competitive Ability of Wushu Routine Athletes[J]. Sports Forum, 2010.

[7] Li T. Affects and Countermeasures of the Implementation of New Rules on College Amateur Wushu Training[J]. Wushu Science, 2012.

[8] Wang X, College W. A Research on the Creation Method of Competitive Wushu Routine(Changquan)[J]. Chinese Wushu, 2014. 